\title{
DEMOCRAZIA E REFERENDUM
}

\author{
di Yannis Papadopoulos
}

\section{Precisazioni concettuali}

Gli strumenti referendari rappresentano un tentativo di conciliare i limiti posti dalle grandi dimensioni del corpo elettorale - che rendono impossibili vere e proprie assemblee popolari del tipo Landsgemeinde - e l'esigenza di una partecipazione diretta dei cittadini ai processi decisionali. Di questi strumenti analizzerò in questa sede $\mathrm{i}$ più «democratici», quelli cioè che sono il prodotto di spinte «dal basso», frutto di richieste promosse da un certo numero di elettori al di fuori di qualsiasi controllo da parte del potere politico ${ }^{1}$. Non terrò conto, pertanto, né dei referendum obbligatori, imposti dalla costituzione (in generale nei casi in cui si tratta di modificarla o di consentire un parziale abbandono di sovranità), né di quelli organizzati a scopi tattici o di legittimazione, né, a fortiori, dei plebisciti a carattere personale ${ }^{2}$.

Strumenti come quelli appena indicati sono assai diffusi, a livello nazionale, in Svizzera, ma anche in Italia (Uleri 1994)

Questo saggio è una versione abbreviata di una relazione presentata al $16^{\circ}$ Congresso mondiale dell'IPSA, Berlino, 21-25 agosto 1994.

1 Tale numero può essere fissato sia in cifra assoluta, sia in proporzione all'elettorato. Una delle dimensioni rilevanti dell'analisi delle funzioni del referendum, sottolineata da Smith (1986), è quella del grado di controllo delle procedure referendarie da parte delle autorità.

2 Neppure i recenti referendum sul Trattato di Maastricht, tenuti in vari paesi europei, corrispondono al modello qui esaminato, dal momento che essi derivavano da esigenze costituzionali oppure sono stati decisi dai governi.

${ }^{3}$ Dal 1970 al 1993 ci sono state in Italia 50 richieste di referendum, mentre in Svizzera, durante lo stesso periodo, il popolo è stato consultato 108 volte in seguito a domande formulate dai cittadini. In Italia non esiste formalmente alcun diritto d'iniziativa popolare. Questo scarto è parzialmente colmato dall'assenza di limiti temporali alla contestazione di una legge attraverso iniziativa referendaria (fissati in Svizzera in 90

RIVISTA ITALIANA DI SCIENZA POLITICA / a. XXV, n. 2, agosto 1995 
così come in numerosi stati americani (Auer 1989; Mény 1978). Fra questi strumenti, mi concentrerò su quelli che vertono sulla valutazione di singole politiche - a proposito dei quali si parla di policy votes (Suksi 1993) -, tralasciando invece istituti che, in qualche misura, si presentano come anti-plebisciti o voti di sfiducia personalizzati (ad esempio l'istituto della «revoca» degli eletti negli Stati Uniti). Allo stesso modo, non mi soffermerò su quegli strumenti che non sono vincolanti per le decisioni, ma semplicemente consultivi, come i vari diritti di petizione.

Definito così il campo di indagine, possiamo identificare due fattispecie, in base ad un'analogia funzionale e malgrado le variazioni che si possono scoprire al loro interno. Si tratta del diritto di veto referendario, che consente a un gruppo di cittadini di domandare che gli elettori si pronuncino su decisioni politiche già prese, e del diritto d'iniziativa popolare, che permette invece a un gruppo di cittadini di fare delle proposte e di sottometterle al corpo elettorale 4 . Nel primo caso possiamo parlare di consultazione deliberativa, nel secondo di consultazione propositiva (Uleri 1994).

Di queste tecniche politiche esamineremo funzioni e disfunzioni, facendo riferimento sia ai «vertici» (la classe politica e l'élite dirigente), sia alla «base» (i comuni cittadini, coloro che rientrano sotto la nozione astratta di popolo). Nel primo caso ci si chiede quale sia il contributo (positivo o negativo) di questi meccanismi alla governabilità; nel secondo quale sia il loro contributo alla partecipazione e alla democratizzazione delle decisioni. Si tratta di procedere, insomma, ad un'analisi degli effetti politici del diritto d'iniziativa e di referendum, aspetto curiosamente trascurato dalla pur abbondante letteratura sui meccanismi referendari.

A costo di essere troppo schematici, si può affermare che, tradizionalmente, l'uso dei meccanismi referendari è percepito dalle autorità, quando da esse non controllato, come una poten-

giorni dalla sua adozione); ma solo in parte, in quanto in Italia un gruppo di cittadini non può domandare che si legiferi in ambiti dove vi sia un deficit normativo (ad esempio nel campo delle nuove tecnologie applicate alla medicina).

${ }^{4}$ Le variazioni interne ai due tipi di meccanismi vertono sulla natura della decisione, sulle modalità di trattamento della domanda da parte del sistema politico (ruolo del parlamento, obbligo o meno di ricorrere in seguito al voto popolare), sulla quantità e le modalità di raccolta delle firme, sull'esistenza di un quorum di partecipazione, sul carattere abrogativo o sospensivo del veto, ecc. Queste variazioni non sono tuttavia sufficienti per influire sulle differenziate funzioni dei due meccanismi referendari. 
ziale perturbazione. Al contrario, tali meccanismi appaiono a coloro che non fanno parte dell'élite politica come una risorsa supplementare di partecipazione agli affari pubblici, un mezzo aggiuntivo per l'esercizio della libertà di espressione, una modalità di riequilibrio dei rapporti di potere. Queste due prospettive, perfettamente simmetriche, sono le più conosciute. L'analisi empirica può però condurre anche a valutazioni non solo diverse, ma fondamentalmente opposte. Possiamo segnatamente concepire i meccanismi referendari come strumenti di integrazione delle opposizioni, funzionali dunque alle élites. Allo stesso modo, essi possono rivelarsi disfunzionali per gli oppositori, favorendo il recupero delle rivendicazioni di questi da parte del sistema.

L'obiettivo di questo lavoro è mettere in evidenza ambivalenza e plurivocità dei meccanismi referendari lasciate di solito in ombra. E significativo del resto, se si considerano le contrapposte argomentazioni dei teorici elitisti della democrazia quali Weber (1968), Schumpeter (1964) e Sartori (1987), e quelle di coloro che vorrebbero favorire un allargamento della partecipazione come Barber (1984), Dahl (1985), Macpherson (1985), Pateman (1970) e Poulantzas (1978), come né l'una né l'altra scuola di pensiero concepisca l'allargamento della partecipazione come funzionale per coloro che dominano e disfunzionale per i dominati. Le due concezioni concordano infatti nel considerare la partecipazione diretta un surplus di democrazia, anche se ciò è criticato dagli uni e ben accolto dagli altri.

Élites politiche e meccanismi referendari: una difficile convivenza

L'argomento principale dei sostenitori della professionalizzazione della politica contro un allargamento della partecipazione verte sull'incompetenza dei non professionisti nella gestione degli affari pubblici. Il lavoro teorico nel quale la tesi dell'incompetenza è avanzata con le minori esitazioni è quello di Schumpeter, che riduce la democrazia a competizione tra élites. Le analisi empiriche relativizzano di solito affermazioni così generalizzanti: sappiamo ad esempio che la competenza, tanto da un punto di vista oggettivo che soggettivo, varia in funzione della complessità delle questioni politiche e del patrimonio culturale degli individui.

Questo argomento è stato tuttavia criticato in altri modi e 
anche sul piano teorico. Possiamo in effetti domandarci perché i cittadini dovrebbero essere considerati sufficientemente competenti per eleggere persone «capaci» che li rappresentano e non per prendere essi stessi delle «buone» decisioni: «se l'opinione degli elettori è irrazionale, non lo è certo meno l'elezione dei rappresentanti» (Habermas 1989, 29). Schumpeter (1964, 350) ammetteva d'altra parte che i parlamentari potevano anche comportarsi nel modo in cui Gustave Le Bon descriveva la psicologia delle folle... Senza giungere a tanto, si può ragionevolmente sostenere che l'essenza delle decisioni politiche sta nel fatto che queste sono fondate su valori, e che anche una politica «illuminata» dal ricorso alla conoscenza scientifica rimarrà irriducibile a quest'ultima (Willke 1992, 285). C'è dunque davvero una frattura insanabile tra il comportamento dei competenti e quello dei profani?

Il punto merita qualche precisazione. Esiste come è noto un dibattito, abbastanza sterile almeno sul piano dell'analisi empirica, fra la tesi che vede la decisione come risultato di orientamenti ideologici e la tesi che vede la decisione come il risultato di calcoli di natura strategica. Se le teorie della «razionalità limitata» hanno cercato di conciliare i due aspetti (razionalità limitata dai valori), mi parrebbe più produttivo pensare che l'approccio varî secondo gli individui, gli oggetti e le circostanze, sebbene gli studi sul comportamento elettorale tendano a mostrare un'evoluzione in direzione del modello dell'individuo utilitarista (Lavau 1986). Cionondimeno, è vero che l'ambiente parlamentare fornisce, rispetto alle campagne referendarie, un terreno strutturalmente più favorevole alle deliberazioni e all'argomentazione. In quella sede si è infatti obbligati a persuadere piuttosto che ad affermare dei dogmi. I parlamentari, inoltre, hanno dei mezzi per fare del logrolling che il cittadino comune non ha: cedere su un argomento di importanza secondaria per sé ma primario per l'altro, a condizione che questi s'impegni a fare altrettanto in futuro, favorisce le contrattazioni e i compromessi. Niente di tutto questo avviene nelle procedure referendarie. Esse si concludono con decisioni binarie: «sì o «no» e costituiscono per di più la fase di chiusura del percorso decisionale: le autorità non hanno niente da offrire ai cittadini in cambio della loro adesione.

L'argomento dell'incompetenza permette d'insistere sul tema della plasmabilità dei cittadini. Essi sarebbero facilmente manipolabili da leaders carismatici o da altri demagoghi populi- 
sti, tanto più oggi in presenza di sofisticate tecniche di marketing politico. Non è tanto il fatto che i cittadini non decidono in autonomia - cosa di per sé già poco conforme all'ideale democratico - a preoccupare, quanto piuttosto che l'assenza di un'etica di autolimitazione e di responsabilità costituisce un serio ostacolo alla governabilità. Essa è causa di sovraccarico (overload) e di «stress» per il sistema politico. I partecipazionisti avrebbero senza dubbio delle buone ragioni per rallegrarsi, ma è Sartori $(1987,122)$ a ricordarci che la crisi di governabilità non è un gioco a somma zero ma un gioco a somma negativa nel quale tutti i contendenti risultano perdenti.

Conviene precisare la nozione di sovraccarico, poiché essa può avere due accezioni diverse, connesse all'uso di strumenti referendari distinti.

Sovraccarico è anzitutto eccesso di inputs, di domande indirizzate al sistema politico (constatazione da cui muove la critica neo-conservatrice alle politiche dello Stato sociale). In questa prospettiva la maggior quantità di offerta alla quale i partiti si affidano per ragioni di competizione elettorale dà luogo a un'inflazione di rivendicazioni che non possono essere soddisfatte dalle autorità. Il risultato è una crisi di legittimità. Si può pensare che i meccanismi referendari aggravino tutto ciò. Se infatti, per ragioni di concorrenza, i partiti evitano di selezionare e di gerarchizzare le domande sociali, ciò è ancor più vero per il meccanismo referendario di iniziativa popolare che mira esplicitamente ad aggirare i filtri di selezione delle domande. Per ragioni elettorali, ad esempio, accade spesso che i partiti promettano, in campagna elettorale, di diminuire le tasse. Ciò può danneggiare la capacità dello Stato in materia di estrazione delle risorse, sebbene una volta al potere gli stessi partiti si vedano poi costretti a dimenticare simili promesse... Ora, non solamente l'iniziativa popolare permette di aggirare i partiti, ma, soprattutto, le sue domande sono vincolanti per le autorità se accettate al momento del voto. Si tratta, dunque, di un elemento supplementare di indebolimento e di permeabilità del sistema politicos.

Ciò che si rimprovera all'iniziativa popolare è di generare una dose d'innovazione che il sistema non è in grado di assimi-

${ }^{5}$ Le autorità politiche svizzere si sono trovate di fronte a questo problema dell'«eccesso» di domande dovuto a una proliferazione d'iniziative. Nel 1977 il numero di firme necessario per dare avvio a un'iniziativa popolare è stato innalzato da $50.000 \mathrm{a}$ 100.000 
lare. L'argomento è capzioso dal momento che mira a tradurre in termini tecnici un problema che in realtà è politico. Il problema, infatti, non è tanto che le proposte corrono a un ritmo talmente rapido che il sistema non ha il tempo né i mezzi per rispondere. $\hat{\mathrm{E}}$ semplicemente che il loro contenuto non concorda con i valori dominanti del sistema. E, tuttavia, bisogna effettivamente convenire che esso crea difficoltà considerevoli alla governabilità. Se la risposta è stata un rafforzamento dei filtri selettivi, ciò è avvenuto perché le autorità si sono trovate di fronte a situazioni di stress: l'iniziativa popolare è un meccanismo che introduce una dose d'incertezza difficilmente tollerabile perché tende a sconvolgere in modo imprevisto l'ordine del giorno dell'agenda politica. A queste condizioni, diviene estremamente disagevole per il sistema politico adempiere al proprio ruolo di «guida». L'argomento non è nuovo, ma merita di essere ripreso. Il punto da fermare è che il meccanismo di iniziativa popolare può contribuire al sovraccarico del sistema politico. È lecito domandarsi, anche se la risposta è ben lungi dall'essere semplice, in quale misura il sistema politico può e deve essere sensibile alle pressioni dell'ambiente: dove si arresta la responsiveness e dove inizia il populismo, con tutto ciò che esso implica in termini di connotazioni negative?

La nozione di sovraccarico può dipendere, in secondo luogo, da un eccesso di contestazione degli outputs, delle decisioni del sistema politico, fenomeno diverso dall'eccesso di inputs e collegato non al meccanismo dell'iniziativa popolare bensì a quello del veto referendario che consente al corpo elettorale di pronunciarsi sulle politiche pubbliche. Il problema, in questo caso, non è più il sovraccarico di domande, ma la paralisi decisionale che può scaturire dal diritto di veto popolare e dall'eventuale annullamento, in un solo giorno, di anni di paziente lavoro legislativo. Ciò che in questo caso preoccupa le élites politiche non sono tanto le pressioni incontrollate verso l'innovazione, ma l'«eccesso» di conservatorismo e la difficoltà a modificare lo status quo ${ }^{6}$.

La possibilità di veto referendario al termine del percorso

${ }^{6}$ La nozione di innovazione è vista qui in senso tecnico, come spostamento o mutamento dallo status quo, a prescindere dal suo colore ideologico: un'iniziativa popolare dal contenuto reazionario rimane, in quest'ottica, un'innovazione; simmetricamente, si parla di conservatorismo quando lo status quo è mantenuto anche se le domande d'innovazione sono ideologicamente meno progressiste! 
decisionale introduce un diverso e ulteriore elemento d'imprevedibilità. La stessa cosa accade con ogni nuovo filtro introdotto in tale processo (ad esempio la necessità di ottenere su un progetto anche il consenso di una seconda camera). Se tuttavia certi sistemi politici hanno moltiplicato le istanze prima di raggiungere l'accordo, questa «ridondanza» non è senza scopo: si tratta di assicurarsi in più riprese e presso pubblici differenti della accettabilità di una decisione, il che peraltro è innegabilmente rassicurante per le élites (Bendor 1985) ${ }^{7}$. Questa prospettiva psicologica, formulata in termini di comunicazione, può essere utilmente completata dalla teoria dei «costi» elaborata dagli economisti della «scelta razionale» (Buchanan e Tullock 1962): nel prendere qualsiasi decisione gli attori devono scegliere tra la riduzione di due tipi di costi, che tuttavia variano secondo un rapporto di proporzionalità inversa, obbligando il decisore a ragionare in termini di trade-offs. Ridurre i costi esterni, vale a dire i disagi inflitti a coloro che non sono stati consultati - un caso tipico è quello dell'inquinamento ambientale ad opera delle industrie - consente di accrescere sostegno e legittimità. Ma ciò non può verificarsi che a prezzo di un aumento dei costi di transazione, cioè di un considerevole investimento in termini di tempo, denaro e sforzi organizzativi nelle consultazioni; va da sé che questi costi crescono con l'aumentare del numero di tappe, di attori e di istanze che intervengono nel processo. È in questo senso che la possibilità di ricorrere al veto referendario implica dei costi, senza menzionare l'incertez$\mathrm{za}$ che introduce in merito alla reazione del pubblico.

La Svizzera costituisce un caso da manuale a questo riguardo, giacché esiste un consenso nella comunità scientifica a proposito del deficit d'innovazione del suo sistema politico, deficit largamente dipendente dall'esistenza del veto referendario. I risultati di un lavoro comparativo sono emblematici: Immergut (1992) constata i continui fallimenti degli sforzi di rendere più sociale la sanità svizzera, in contrasto con l'esperienza francese della Quinta Repubblica e con quella svedese. Ora, la persistenza della linea ultra-liberale in materia di politica sanitaria in Svizzera non può essere spiegata, secondo l'autore, né con la caratterizzazione particolarmente conservatrice del governo -

\footnotetext{
7 E accessoriamente anche per le minoranze, che beneficiano così di molteplici sedi di intervento.
} 
certo reale, ma nel caso specifico i partiti di destra erano favorevoli alle riforme - né con la posizione privilegiata delle associazioni dei medici o quella subordinata dei sindacati. La spiegazione vera resta il veto referendario.

L'effetto conservatore del referendum è tale, in Svizzera, che spesso il solo minacciarlo basta a dissuadere i promotori di una qualche innovazione ad andare avanti nel loro progetto. Gli attori reputati dalle autorità come capaci di lanciare un referendum e di vincerlo godono di un vero e proprio potere di veto preventivo che fa sì che le loro obiezioni siano prese sul serio. Un tale potere non è più inteso come capacità di prendere delle decisioni, ma come capacità di impedire che esse siano prese, conformemente al celebre approccio della «non-decisione» di Bachrach e Baratz (1962). E dunque sufficiente, in questa ottica, che gli attori annuncino la loro intenzione di lanciare un referendum perché le loro domande siano prese in considerazione oppure che un progetto da essi malreputato venga silurato. Questo «potenziale di ricatto» di cui sembrano disporre certi potenti gruppi nella vita politica elvetica si trova fortemente limitato in Italia dalla minaccia dello scioglimento anticipato del parlamento, che è un potente mezzo di pressione nei confronti dei promotori potenziali di referendum. I partiti, d'altronde, hanno fatto pressioni sulla Corte costituzionale, la quale può invalidare le domande di referendum e costituisce un filtro cruciale (16 domande respinte sulle 50 formulate dal 1970 al 1993).

Il concetto di «potenziale di ricatto», che varia come abbiamo appena visto a seconda dei filtri istituzionali esistenti, merita qualche ulteriore considerazione. Per Crozier e Friedberg $(1981,168)$ il potenziale di ricatto è funzione della fonte d'incertezza che un attore è in grado di controllare. Quanto più la cooperazione di un attore è indispensabile per ridurre l'incertezza ambientale, e quanto minori sono le possibilità di ricorrere a un concorrente per gli stessi «servizi», tanto più tale attore possiede i mezzi per ricattare il sistema. Il concetto è stato utilizzato anche da Sartori nella sua opera classica sui sistemi partitici $(1976,123$ ss.), ma in una prospettiva abbastanza diversa. Per lui il «potenziale di ricatto» è quanto resta ai partiti estremisti definiti anti-sistema e privi di «potenziale di coalizione», cioè della possibilità di accedere al governo. Non resta loro che un «potere di veto» da usare al momento della deliberazione delle leggi, il che costringe i partiti al potere a tener conto delle 
loro domande e a frenare il loro cammino verso la moderazione e l'assunzione di posizioni centriste. Nel nostro ambito, i due «potenziali» possono essere riuniti: in Svizzera è proprio grazie al fatto che i conservatori cattolici, poi gli agrari e infine i socialisti hanno maneggiato con successo il veto referendario contro la legislazione che essi sono stati gradualmente cooptati nel governo, in modo da ridurre le incertezze circa l'accoglimento delle politiche statali. Il potenziale di ricatto si è dunque tradotto in potenziale di coalizione. Di fronte al potenziale di ricatto di determinati attori, il sistema politico dispone di due soluzioni: adattare i suoi programmi d'intervento, ma anche integrare gli oppositori, sperando di socializzarli ai propri obiettivi e di limitare così i mutamenti di rotta. Questa seconda soluzione è l'opzione della cooptazione, che nei lavori di Michels sull'integrazione delle élites dei partiti operai ha trovato la sua più classica enunciazione. Altri autori hanno evocato l'idea dell'ostruzionismo contro le politiche pubbliche, di cui il diritto di referendum costituisce una risorsa supplementare. $\grave{\mathrm{E}}$, per esempio, il caso della teoria della «dipendenza strutturale» delle nostre società nei riguardi dell'economia privata e dei capitalisti, i quali dispongono di una possibilità di «defezione» (exit) - fuga di capitali, riduzione degli investimenti, ecc. - che colpisce la società nel suo complesso nella forma, ad esempio, di una perdita di posti di lavoro o di redditi statali necessari per finanziare le politiche sociali (Offe 1984). Questa fonte d'incertezza non può essere ignorata dalle autorità $\mathrm{e}$ riduce il loro margine di manovra. Il diritto di referendum è una possibilità di «protesta» (voice) complementare alla «defezione»: si caratterizza anch'essa come limite al volontarismo dei governanti. Il veto referendario è dunque un fattore di rigidità supplementare nelle mani di gruppi che, secondo la teoria di Olson (1982), agiscono in difesa di interessi particolaristici. Quanto maggiore sarà la forza di tali interessi - è il caso di società come le nostre, in cui le spinte all'autorganizzazione degli interessi particolaristici sono più forti di quelle relative alla difesa di interessi generali - tanto più le autorità si troveranno a fronteggiare gli sbarramenti eretti dai gruppi contro mutamenti che vanno a colpire i loro «diritti acquisiti». In quest'ottica, i gruppi organizzati sono un fattore di perturbazione rilevante nella formazione delle politiche pubbliche. Una prospettiva comparata pare confermare questa tesi: in occasione dei negoziati sullo spazio economico europeo, l'Austria e i paesi nordici hanno manifestato una volontà molto 
più ferma della Svizzera nell'assicurare una liberalizzazione dei settori economici protetti (Sciarini 1992, 316). La differenza può essere spiegata col potere di veto referendario in mano a gruppi di pressione protezionisti in questo paese.

In termini più generali, in Svizzera il diritto di referendum popolare ha portato allo sviluppo di una democrazia del negoziato e, quindi, alla consultazione degli interessi prima che qualsiasi progetto di legge venga discusso in parlamento ${ }^{8}$. Va da sé che ad essere presi in considerazione sono soprattutto gli argomenti degli attori dotati di «capacità referendarie» (referendumsfähig). E proprio la minaccia del referendum, più che la sua effettiva realizzazione, il fattore di rigidità. E questa minaccia pesa come una spada di Damocle sull'insieme dei processi decisionali, ivi compresi i lavori delle «commissioni di esperti» (Germann et al. 1985, 185). Il referendum diventa quindi uno «strumento di pressione» e di ricatto. Non a caso, dal 1874 ad oggi - da quando cioè il referendum è stato accolto nella Costituzione federale - soltanto il $7 \%$ delle decisioni ne è stato fatto oggetto. Come ricorda Aubert i referendum più efficaci sono infatti quelli che non sono stati tenuti: la richiesta di un referendum contro una legge è uno scacco alla democrazia del negoziato.

Queste constatazioni empiriche richiamano il legame pernicioso tra consenso e conservatorismo messo in evidenza da Scharpf $(1991,60)$ : la necessità del consenso finisce infatti per favorire lo status quo, dal momento che l'assunzione di decisioni consensuali permette agli attori di difendere l'allocazione delle risorse esistenti e impedisce ogni redistribuzione delle stesse. La minaccia del veto referendario e l'ampliamento del numero degli attori consultati finisce così col privilegiare chi dispone del potenziale di ostruzionismo e di ricatto più elevato ed è in grado di minacciare ritorsioni in caso di mutamenti dello status quo. La funzione dei referendum diventa quindi più

${ }^{8}$ La tesi di Neidhart (1970) è forse un po' troppo condizionata dal caso svizzero. Il diritto di referendum spinge infatti al negoziato semplicemente perché accresce il potenziale di ricatto di certi attori. Neanche per la Svizzera si tratta però della ragione principale. Lijphart (1977) ha mostrato ad esempio che giungere a decisioni attraverso accordi di vertice fra le élites delle principali sub-culture è una modalità che consente di evitare l'esplosione delle società segmentate. E Katzenstein (1985) sostiene che i meccanismi di concertazione sono particolarmente sviluppati nei piccoli paesi, dove, in presenza di una forte dipendenza dagli scambi con l'estero, lo «stringersi insieme» permette almeno di limitare l'incertezza interna. 
comprensibile se la si esamina dal punto di vista del contributo alla gestione della complessità delle nostre società. La risposta istituzionale alla complessità dell'ambiente sociale sembra essere una corrispondente «complessificazione» dello stesso sistema politico e, più precisamente, la moltiplicazione delle istanze e dei livelli di decisione pertinenti (Vielschichtigkeit): consultazione di una pluralità di attori, federalismo e bicameralismo, decentramento, ecc. Ciò corrisponde alla legge della «varietà richiesta» di Ashby (1956, 202 ss.), ben nota all'analisi dei sistemi: per adattarsi e sopravvivere allo stress provocato dalle «aggressioni» del suo ambiente, un sistema deve essere almeno altrettanto complesso. I fallimenti degli esempi contrari sono d'altronde evidenti: poiché il federalismo sovietico non era che una cortina fumogena, l'impero dell'URSS è crollato sotto il peso dei diversi nazionalismi; ed è per non avere integrato la componente islamica nella società che lo Scià dell'Iran è stato rovesciato da una rivoluzione. Il sistema politico deve quindi aprirsi per integrare la complessità sociale. L'apertura cessa così di presentarsi come misura di democraticità - così come la intendono i teorici partecipazionisti - per divenire invece un imperativo della governabilità.

Il sistema, tuttavia, non può essere reso complesso all'infinito senza rischi di sovraccarico e di paralisi decisionale; può integrare una dose limitata di complessità, altrimenti ne viene pervaso. A tale riguardo $\mathrm{i}$ blocchi indotti dal veto referendario sono significativi: la gestione della complessità implica un aumento dei «poteri positivi» (Zolo 1992, 60); l'istituzione del veto referendario favorisce al contrario la proliferazione dei «poteri negativi», indebolendo la capacità di direzione e di guida del sistema politico. Il referendum popolare pone quindi la questione dell'auto-limitazione e delle risorse morali degli attori politici (Offe 1989): la tentazione di ricorrervi per difendere $i$ propri punti di vista particolari è grande, e si combina con una assenza totale di empatia tanto per l'interesse pubblico, quanto, supponendo che quest'ultimo sia un'astrazione, per i punti di vista particolari degli altri. La complessità esige la messa a punto di meccanismi che favoriscano, e non che inibiscano, lo sviluppo di questa «cultura dell'empatia».

Più elevato è il numero di attori e delle tappe del processo decisionale - e fra queste i meccanismi referendari -, più pressante diventa l'esigenza di cooperazione fra le parti allo scopo di ridurre i costi di transazione accresciuti dall'aumento dei pas- 
saggi intermedi. Si è visto però che la logica dei comportamenti popolari non è analoga a quella delle élites. L'applicazione di una qualsiasi logica di gratificazioni differite - su cui si fonda, per esempio, la cooperazione tra sindacati e imprenditori negli assetti di tipo neo-corporativo (concessioni sindacali in materia di rivendicazioni salariali in cambio di una garanzia sul mantenimento futuro dei livelli di occupazione) - si rende difficile a livello di massa. L'assenza di incitazioni alla «cooperazione» popolare - di uno spirito di cooperazione che favorisce la prevedibilità dei comportamenti $i^{9}$ - contribuisce a introdurre una ulteriore dose di imprevedibilità nei processi decisionali.

Se gli scambi tra le élites sono spesso viziati da illegittimità e considerati come «transazioni collusive» (Dobry 1986), ciò non impedisce che essi favoriscano tuttavia la stabilità di un sistema. Bisogna pur ammettere che una dose di elitismo e di modi poco democratici sono inevitabili quando l'obiettivo principale è quello di assicurare i compromessi necessari alla gestione della complessità. In tal senso si può manifestare una seria antinomia tra le esigenze di gestione della complessità e di guida della società, da una parte, e quelle di democratizzazione dei processi decisionali, dall'altra ${ }^{10}$.

Fin qui si sono passati in rassegna i principali elementi che fanno sì che i meccanismi referendari, quando non sono controllati dalle autorità, possano divenire fonte di perturbazione e rendere più difficile l'elaborazione di soluzioni adeguate per risolvere i problemi. Resta ora da mostrare in che modo i meccanismi referendari, contrariamente alla visione prevalente, presentino anche dei lati funzionali per la parte «alta» del sistema politico.

9 E il caso di ribadire che un tale «spirito» non è affatto il risultato delle predisposizioni psicologiche degli attori, ma dipende dalle concrete procedure e regole del gioco.

10 Sappiamo, per esempio, che gli accordi di vertice tra imprenditori e sindacati indispensabili ad una gestione più efficace della crisi (Schmidt 1982) - hanno avuto un migliore effetto in quei paesi dove le organizzazioni erano fortemente centralizzate e fruivano di un monopolio della rappresentanza che vanificava la «fuga» di una base insoddisfatta verso la concorrenza. A contrario, Scharpf (1990) ha mostrato che i cattivi risultati dell'economia britannica durante la crisi degli anni '70 si spiegano con la struttura decentrata del movimento sindacale e con l'atteggiamento intransigente dei capi delle commissioni interne al livello d'impresa; queste due condizioni hanno reso impossibile il raggiungimento di un «contratto sociale» al vertice. In tal modo, la debolezza delle strutture verticali all'interno del movimento sindacale ha condotto a risultati tutt'altro che ottimali per tutti (inflazione, disoccupazione). 
Legittimazione e integrazione

Considerare gli strumenti referendari come elemento di perturbazione impedisce di prenderne in considerazione la dimensione legittimante. Anche entro una concezione strumentale e pragmatica della legittimità - e quindi non solo auspicabile in rapporto all'ideale democratico, ma funzionale alle esigenze delle autorità politiche - l'opportunità di avviare un referendum contro una determinata decisione offre un surplus non trascurabile. Gli ingegneri istituzionali, d'altra parte, non hanno rinunciato a immaginare tipi diversi di correttivi, anch'essi in grado di legittimare ex post le decisioni assunte o di stimolare ex ante la moderazione: si pensi per esempio, nei paesi in cui non esiste la possibilità del veto referendario, al controllo di costituzionalità delle leggi. Quando una decisione ha superato con successo l'ostacolo referendario abbiamo però un indicatore ulteriore di accettazione non limitato alla sola sfera delle élites parlamentari. E ciò indebolisce le critiche della minoranza, giacché il popolo sovrano ha già deciso in ultima istanza.

Si è già visto che in Svizzera non è questo lo scenario usuale, poiché il referendum è richiesto solo molto raramente. Tuttavia se un gruppo si tuffa nella battaglia, senza arrivare a raccogliere le firme necessarie, se ne può ricavare un segno della volontà popolare. Non volere o non riuscire a cogliere l'opportunità del referendum è anch'esso un modo per legittimare le decisioni delle autorità, o per rassicurarle circa l'assennatezza delle loro scelte.

Sappiamo inoltre che la minaccia del referendum può contribuire allo sviluppo di consultazioni e negoziati assai prima che un progetto si trasformi in legge vera e propria. Fin qui si è posto l'accento sull'appesantimento del processo decisionale che sembra derivarne. Lo sviluppo di meccanismi di concertazione consente però anche di ridurre le manifestazioni di reazione alle decisioni e, quindi, anche l'insoddisfazione e la protesta.

La consultazione degli interessati al fine di evitare il referendum può essere assimilata ad una simulazione delle reazioni della società civile verso un progetto statale. Tale simulazione non sarà certo perfetta. Si basa infatti su due postulati piuttosto azzardati: che la società sia «rappresentata» dagli attori collettivi consultati e che le istanze consultate siano rappresentative della base. Si tratta, tuttavia, di una simulazione tutt'altro che trascurabile come mezzo per ridurre l'incertezza. La con- 
sultazione degli attori in merito a un progetto è fruttuosamente comparabile a un «colpo a vuoto» che suscita un feedback o una correzione anticipata, prima cioè che un progetto pubblico si sia concretato in una decisione vera e propria che, in caso di «errore», avrebbe conseguenze ben più gravi sul piano delle reazioni sociali (Ossipow 1988).

Nell'estate del 1992 le strade francesi sono state bloccate dalla rivolta «selvaggia» dei camionisti ostili all'introduzione della patente a punti. In Svizzera lo scenario sarebbe stato ben diverso: Charles Friderici, deputato liberale del cantone di Vaud e lui stesso grosso trasportatore, dichiarò che contro una tale regolamentazione sarebbe stata fatta richiesta di referendum... Se è bene, dunque, essere consapevoli dei rischi di blocco preventivo inerenti a tale possibilità, possiamo tuttavia apprezzarne gli effetti positivi sulla pace sociale. Più in generale, il rischio di referendum invita alla prudenza, a non cedere a illusioni volontaristiche e all'attivismo, a non sopravvalutare le capacità d'intervento del sistema politico nei confronti degli altri sistemi sociali. Si potrebbero citare molti esempi di fallimenti che i meccanismi referendari avrebbero permesso di attutire, essendo senza dubbio meno imbarazzante per i dirigenti il risultato negativo di una consultazione popolare rispetto a eventuali rivolte e manifestazioni violente, ed essendo preferibile una consultazione negativa ad una sconfitta decretata dalla piazza. La Francia avrebbe senza dubbio potuto evitare di arrabattarsi nel corso dei numerosi tentativi contraddittori di regolare scuola e università, a proposito dei quali vari gruppi sociali hanno poi colto l'occasione per «votare con i piedi» e andare in piazza. Ancora di recente i consensi verso il primo ministro Balladur si sono sensibilmente abbassati in seguito alle violente manifestazioni degli studenti contro il progetto di «contratto d'inserimento professionale», che permetteva una qualche forma di dumping salariale in cambio della possibilità d'inserimento nel mercato del lavoro. E nel Regno Unito la signora Thatcher ha perso il posto di primo ministro per avere ignorato le reazioni suscitate dalla poll tax, mentre un regime meno centrato sui poteri del primo ministro e più aperto di quello maggioritario britannico ai feedbacks popolari sarebbe stato verosimilmente costretto ad una maggiore circospezione.

Il secondo effetto che scaturisce dalla possibilità del veto referendario - nonché dall'opportunità di iniziativa legislativa popolare - è quello dell'integrazione delle opposizioni. Se la Svizze- 
ra è «un caso paradigmatico di integrazione politica» (Deutsch 1976) lo deve anche agli effetti delle sue istituzioni referendarie. Se si pensa al repertorio delle azioni dei movimenti di protesta, il referendum appare un mezzo sostitutivo dell'espressione violenta delle rivendicazioni. Si potrebbe ipotizzare che in assenza di canali legali di comunicazione tra ambiente e sistema quali il diritto di referendum e quello di iniziativa, le diverse sensibilità si esprimerebbero in modo più radicale se si sapessero minoritarie in parlamento. Le istituzioni referendarie svolgono dunque una funzione tribunizia (Lavau 1969, 18). La funzione tribunizia è svolta a vantaggio di coloro che si sentono esclusi o emarginati dai processi decisionali. Consiste, dunque, nel canalizzare e integrare le opposizioni favorendone la libera espressione. Proprio la possibilità di un'espressione istituzionalizzata per via referendaria ha favorito, in Svizzera, una canalizzazione delle opposizioni sia attraverso la cooptazione degli oppositori, sia attraverso la loro associazione al processo decisionale. Va precisato che non si tratta di una «legge ferrea» e che il determinismo istituzionale ha i suoi limiti: negli Stati Uniti, per esempio, i meccanismi referendari non hanno alimentato l'accordo fra gli attori.

I processi referendari possono essere considerati anche come dei tentativi di socializzare i destinatari delle politiche agli obiettivi del sistema, ovvero come meccanismi di prevenzione del conflitto e di costruzione del consenso. Senza scivolare verso discorsi semplicistici, possiamo constatare l'esistenza di una relazione dialettica tra la perturbazione avvertita dalle élites in occasioni partecipative quali i referendum è gli effetti di integrazione che invece disinnescano il radicalismo di opposizioni che hanno l'occasione di esprimersi pubblicamente. E inoltre difficile immaginare che una democrazia sia governabile in modo efficace soltanto sulla base del principio maggioritario, il quale consiste nell'ignorare le domande delle minoranze parlamentari, quando queste ultime dispongono dell'arma referendaria. Tale strumento consente infatti un'ostruzione costante al lavoro del governo e spinge così alla ricerca di compromessi.

\section{Un'opportunità politica supplementare per la «base»}

L'analisi dei movimenti sociali e del loro successo passa spesso per la valutazione della «struttura delle opportunità» 
(Tarrow 1988) a loro disposizione: legge elettorale, sostegno partitico, ecc. I meccanismi referendari possono essere esaminati anche sotto questo profilo, a prima vista come risorsa rilevante per la mobilitazione dal «basso».

Il contraltare delle perturbazioni causate dall'imprevedibilità indotta da questi meccanismi è l'introduzione di una certa dose di varietà nei processi decisionali. La felice formula della democrazia diretta come «sistema politico dalle porte aperte» (Delley 1978) potrebbe così essere completata: dall'alto, le «porte» lasciano entrare «correnti d'aria» capaci di «grippare» il sistema; dal basso, consentono «boccate d'ossigeno» che l'alimentano. Questo intervento popolare, o anche, come si è visto, la sua semplice minaccia, stimola la responsiveness dei governanti e rende visibili i fenomeni di crisi della rappresentanza, lo scarto di preferenze tra governanti e governati. È il caso dei processi di integrazione europea: in Francia, per esempio, l' $89 \%$ dei parlamentari era a favore del trattato sull'Unione europea, mentre l'elettorato si è rivelato profondamente diviso; in Svizzera, quasi i $2 / 3$ dei membri della Camera del popolo (Consiglio nazionale) erano favorevoli al trattato sullo Spazio economico europeo, che però è stato respinto da una ristretta maggioranza popolare. La crisi di rappresentanza resta un aspetto trascurato nelle analisi dei teorici elitisti, sia nelle pagine che Weber dedica alla democrazia diretta in Economia e società, sia in quelle di Schumpeter sulla competizione nella selezione dei dirigenti in Capitalismo, socialismo e democrazia.

Fra gli strumenti referendari, l'iniziativa popolare è senza dubbio il più importante ai fini di un incoraggiamento del pluralismo. Anzitutto, rispetto al diritto di veto referendario, che presuppone una decisione delle élites politiche, il grado di controllo da parte delle autorità è minore in quanto l'iniziativa popolare equivale a una spinta dall'esterno, inserendo nell'agenda politica un problema trascurato o schivato dai governanti. Questo diritto permette di superare gli ostacoli eretti dai gatekeepers del sistema nella selezione delle domande, e di contrastare le tendenze alla chiusura dovute alla dislocazione del potere verso istanze che non godono di legittimità democratica, quali l'amministrazione pubblica. Il meccanismo dell'iniziativa popolare consente agli outsiders «di infrangere il monopolio della rappresentanza detenuto dai partiti e dalle grandi organizzazioni d'interessi; offre la possibilità di modificare l'ordine del giorno politico e di mutare l'ordine delle priorità; suscita la creazio- 
ne di oggetti politici, ovvero la trasformazione in posta in gioco politicamente rilevante di problemi che partiti e organizzazioni lasciano in ombra per mancanza di tempo, per disinteresse o per volontà deliberata» (Delley 1978, 23). «L'iniziativa popolare è un mezzo privilegiato per forzare lo sbarramento corporativo ed evitare il filtraggio delle domande ad opera degli interessi organizzati» (ibidem, 126).

In secondo luogo, al contrario del veto referendario, l'iniziativa popolare non blocca un'innovazione, ma mira a formulare nuove proposte e produce un impulso costruttivo. Pertanto il diritto d'iniziativa estende lo spettro delle opzioni a disposizione per la decisione. E un acceleratore di riforme, che consente di introdurre nel dibattito politico degli argomenti-tabù ${ }^{11}$.

Questo meccanismo referendario permette di riequilibrare le risorse a vantaggio degli attori marginalizzati. Funziona come un rimedio alle asimmetrie nella rappresentanza dei diversi interessi tipiche delle nostre società, e ai loro squilibrati legami col potere politico. È dunque uno strumento di democratizzazione dei processi decisionali che incentiva una logica di «pluralismo egualitario» (Cohen e Rogers 1992). Attraverso questo meccanismo si fornisce un'occasione di aggiramento del monopolio dell'input legislativo detenuto dagli interessi organizzati. In Svizzera, non a caso, gli attori che fanno uso del diritto d'iniziativa sono soprattutto partiti di opposizione, di destra e di sinistra, e organizzazioni ambientaliste (App 1987, 194). Gli strumenti referendari favoriscono inoltre un riequilibrio del potere all'interno delle organizzazioni: i dirigenti devono aver cura della loro base perché hanno bisogno della disponibilità di questa per impegnarsi nelle campagne referendarie (Delley 1978).

Come effetto positivo secondario si può anche sottolineare il ruolo formativo dei meccanismi referendari nei confronti dell'opinione pubblica. Le campagne referendarie contribuiscono alla formazione dello spazio pubblico e sono suscettibili di migliorare il livello d'informazione dei cittadini verso i problemi politici. Secondo i fautori della partecipazione, ciò non può che andare a vantaggio della crescita del sentimento di efficacia politica, con una conseguente crescita anche dell'interesse per

11 In Svizzera il diritto d'iniziativa popolare ha rimesso in discussione sia la presenza degli stranieri sul territorio nazionale, sia l'esistenza dell'esercito. Significativamente, tali questioni hanno suscitato tassi record di partecipazione! 
la partecipazione agli affari pubblici. Si entrerebbe così in un circolo virtuoso, nel quale partecipazione suscita partecipazione. In realtà l'accertamento empirico mostra che il sentimento di efficacia politica dipende in fin dei conti da fattori socio-culturali (livello d'istruzione, reddito, appartenenza socio-professionale, grado di integrazione, ecc.), sui quali l'ingegneria istituzionale non ha tutto sommato che un'influenza limitata (Gaxie 1978). Le ricerche comparate, del resto, falsificano l'idea che il livello d'informazione politica dei cittadini sia superiore nei paesi dove sussistono meccanismi referendari, e che essi incoraggino altre forme di partecipazione (Ranney 1994, 38). Questo mi consente di collegarmi a un aspetto più oscuro e meno noto di questi meccanismi, sempre guardando da un'ottica dal «basso»: potrebbe forse trattarsi, più che di una risorsa della struttura delle opportunità, di un ostacolo che limita i margini d'azione?

\section{La democrazia non è forse dove si pensava...}

Fino ad ora la critica che è stata rivolta ai meccanismi referendari può essere qualificata come di origine elitista, nel senso che si è posto prevalentemente l'accento sulle disfunzioni che i meccanismi referendari possono creare al lavoro dei governanti. Nelle pagine che seguono assumeremo una diversa prospettiva, esaminando i limiti di tali meccanismi dal punto di vista del loro carattere democratico.

Si è appena detto del contributo dei meccanismi referendari all'integrazione delle opposizioni. Visto dal basso ${ }^{12}$, ciò significa anche che l'opportunità offerta ai movimenti sociali di esprimersi attraverso il canale referendario conduce in un certo qual modo al loro esaurimento. Il loro calendario viene ad essere scandito dalle scadenze referendarie, le loro domande si frammentano e si atomizzano a danno delle visioni alternative globali e a lungo termine. Le organizzazioni mobilitano e esauriscono le loro risorse umane e finanziarie in una specie di «ergoterapia democratica» che riduce il loro repertorio d'azione e impedisce di pensare a strategie diverse e forse più produttive ${ }^{13}$. Questa

\footnotetext{
${ }_{12}$ Questo passaggio deve molto al lavoro di Epple-Gass (1991), fondato su una solida conoscenza delle strategie del movimento pacifista in Svizzera.

${ }^{13}$ In occasione di un colloquio con un responsabile delle associazioni studente-
} 
tesi è contestata da chi ammette che le strategie referendarie sono poco utilizzate dai movimenti in ragione del loro costo, ma valorizza l'occasione da esse fornite di mobilitare un largo pubblico e di lanciare azioni parallele (Giugni 1991). Analogamente, Kriesi (1994b) constata che, se nell'insieme il repertorio d'azione dei movimenti sociali in Svizzera è moderato, il ricorso alla democrazia diretta non esclude l'uso anche di altri mezzi d'azione. Il ruolo delle strategie referendarie resta ambiguo: da un lato vincolano l'attività dei movimenti, dall'altro rivestono per tali movimenti un'utilità del tutto relativa! La frequenza del ricorso agli strumenti referendari da parte dei nuovi movimenti sociali sembra inoltre non variare nel tempo (Giugni e Kriesi 1990, 91-93). Se ne può dedurre l'inelasticità di questi strumenti in rapporto alla congiuntura e, dunque, anche una scarsa flessibilità in relazione ai bisogni stessi dei movimenti.

La moderazione delle forme d'azione indotta dai meccanismi referendari comporterebbe anche una deradicalizzazione delle domande (Epple-Gass 1991), necessaria per avere ragionevoli possibilità di ottenere la maggioranza popolare al voto o la speranza di provocare una reazione positiva delle autorità ${ }^{14}$. La democrazia diretta inciterebbe dunque al conformismo e a una trasformazione in senso «convenzionale» dei movimenti alternativi. Le campagne referendarie sembra inoltre alimentino, per ragioni di efficacia organizzativa, la burocratizzazione e la centralizzazione dei movimenti alternativi. La democrazia diretta finirebbe così per rafforzare la componente elitista al loro interno ${ }^{15}$ !

Malgrado il largo pubblico che ne è beneficiario, la decisione referendaria è, infine, lungi dal soddisfare le condizioni di un vero e proprio processo deliberativo come quello prescritto dal cosiddetto modello «pragmatico» di Habermas (1973), fondato sulla partecipazione degli esperti e del pubblico dei citta-

sche elvetiche, mi è stato confermato che il bilancio della loro organizzazione principale, così come gli sforzi dei suoi membri, erano stati completamente assorbiti, nel corso di un lungo periodo, da una raccolta di firme per una iniziativa popolare che alla fine non è decollata!

${ }^{14}$ In Svizzera, i promotori di un'iniziativa popolare possono ritirarla. Ciò accade puntualmente quando essi ritengono che, in un modo o nell'altro, le autorità abbiano più o meno tenuto conto delle loro domande. Il diritto di iniziativa è pertanto anche uno strumento di pressione e di contrattazione.

${ }_{15}$ Delley (1978) sosteneva il contrario. La ricerca empirica dovrebbe maggiormente indagare l'impatto degli strumenti referendari sui movimenti sociali. 
dini alle decisioni del mondo politico. In particolare non si dà un'uguaglianza di risorse fra i partecipanti (tornerò su questo problema), condizione necessaria per una comunicazione immune da fenomeni di potere, nonché per ottenere l'adesione e l'acquisizione del consenso con il ricorso ad argomenti esclusivamente razionali. La realtà della democrazia referendaria resta così molto lontana dall'ideale: «la sovranità del cittadino sarebbe esattamente come quella del consumatore: scegliere un prodotto ignorandone la maggior parte delle caratteristiche, ma subendone fin troppo la seduzione aggressiva» (Andreani 1990, 39). In questa prospettiva lo spazio referendario non sarebbe altro che un mercato fra i tanti, nel quale il libero arbitrio e il margine di scelta del cittadino, a cui la realtà dell'offerta politica appare sfuggente, sarebbero ridotti a zero.

Tutto ciò reca offesa all'ideale dell'individuo aufgeklärt previsto dai principi del funzionamento democratico ereditati dall'Illuminismo. Tenendo ben presente che le competenze decisionali del cittadino variano a seconda del suo patrimonio culturale e della complessità dell'oggetto da trattare, si potrebbe sostenere che in regime di democrazia referendaria - a differenza dei dibattiti parlamentari dove, malgrado le contrattazioni, lo scambio di opinioni resta importante - le deliberazioni sarebbero effettivamente ridotte al minimo. Bisogna tener conto peraltro che la deliberazione è per sua natura limitata agli spazi intermedi delle assemblee e dei gruppi più piccoli: sarebbe impossibile sia nei casi di decisioni autocratiche, sia in quelli di decisioni prese dalla moltitudine ${ }^{16}$. D'altra parte, gli elettori determinano privatamente la loro scelta e ciò tende ad aggravarsi con la mediatizzazione della politica. In tal senso, le attuali pratiche di «tele-democrazia», presentate come l'ultima trovata partecipativa per i cittadini (Saris 1991), non presentano minori ambiguità. Ed è significativo che un leader populista come Ross Perot ne abbia fatto uso negli Stati Uniti come strumento di seduzione. Non si tratta tanto del fatto che tali pratiche suggeriscano la pericolosa idea che il cittadino possa decidere contentandosi di premere un pulsante seduto nel suo salotto, né del fatto che ciò condurrebbe ad una semplificazione estrema delle scelte politiche e che la gente potrebbe decidere su tutto senza disporre delle informazioni adeguate. Il problema fondamentale

16 Traggo questa osservazione dall'intervento di Pasquale Pasquino al seminario su «Sovranità e democrazia referendaria», Università di Losanna, 16 giugno 1993. 
di questo nuovo genere di sondaggi a grandezza naturale consiste soprattutto, mi pare, nell'illusione di onnipotenza che essi alimentano e, ancor peggio, nella banalizzazione dell'atto civico allo scopo di favorire la partecipazione.

Corollario della Vermarktung dello spazio pubblico finisce per essere la colonizzazione delle campagne referendarie da parte delle organizzazioni dotate delle risorse più imponenti. Quando giunge il momento per i cittadini di decidere su una qualche posta in gioco politica, la ben nota formula di Stein Rokkan ci ricorda che «i voti contano, ma le risorse decidono». Questa idea dell'impatto delle risorse mobilitate sull'esito del voto è troppo importante e merita qualche ulteriore chiarificazione. Per cominciare, si può prendere in considerazione l'impatto delle risorse in senso stretto, cioè il denaro. Dato il costo delle campagne referendarie, la domanda è se il risultato sia in qualche misura suscettibile di essere comprato. In caso affermativo sorgerebbero seri problemi: non solo i meccanismi referendari non terrebbero fede all'impegno di riequilibrio delle diseguaglianze politiche a vantaggio dei più deboli, ma fornirebbero ai più forti un'occasione in più per affermarsi. Correlativamente, ogni imprevedibilità scomparirebbe perché basterebbe sapere chi ha investito di più per prevedere il risultato. Per fortuna le cose non sembrano così semplici. In Svizzera, ricerche basate su dati di sondaggio hanno mostrato che «comprare» il risultato di un voto popolare è tutto sommato concepibile (Hertig 1982), giacché di regola prevale l'orientamento in grado di assicurarsi una maggiore presenza negli spazi pubblicitari. Queste risultanze non sono però conclusive: gli indicatori utilizzati da Hertig sono abbastanza grezzi, né si hanno strumenti per conoscere in quale misura abbiano giocato altri fattori $(\mathrm{Pa}$ padopoulos 1994, 148-149). Si aggiunga poi che in presenza di un terreno ideologico fertile e di un retroterra culturale conveniente, gli outsiders hanno qualche possibilità: per esempio, nei casi dei referendum, praticamente la metà dei progetti governativi ai quali non si opponevano che partiti marginali è stata rigettata dall'elettorato (Papadopoulos 1991). Quanto ai risultati delle ricerche compiute negli Stati Uniti, essi non sono privi di contraddizioni (Auer 1989; Cronin 1989), al punto che Magleby $(1994,90)$ si chiede se il fenomeno referendario rappresenti ancora gli interessi dei cittadini, o se esso non sia divenuto un'industria che si auto-alimenta.

Altre risorse utili per procacciarsi adesioni possono essere 
ideologiche e retoriche. Anche in questo caso la diffidenza di certi teorici verso la mancanza di competenze politiche dei cittadini è il rischio della loro manipolazione, questa volta visto dal «basso». I meccanismi referendari si presterebbero bene a un trattamento di riduzione della complessità dei problemi, a slogan semplicistici e manichei: è l'ambiguità della «deprofessionalizzazione» delle scelte politiche (Neidhart 1983, 28). Se così fosse, la democrazia diretta porterebbe rapidamente al rafforzamento del potere oligarchico dei gruppi, una delle promesse mancate delle democrazie secondo Norberto Bobbio (1984) e uno dei rischi di deriva della democrazia immediata verso il potere dei notabili indicati già da Max Weber. Anche su questo punto sarebbe opportuno far ricorso a dati empirici (sappiamo ad esempio che l'attitudine decisionale varia a seconda del livello d'istruzione e del tipo di posta in gioco). L'obiezione merita però di essere presa in considerazione se non si vuole cedere al mito della democrazia diretta.

Se i cittadini fossero totalmente dipendenti nell'assumere le loro posizioni, dovremmo aspettarci una maggiore conformità dei loro comportamenti alle indicazioni delle organizzazioni alle quali si sentono più vicini, essendo le «consegne» ideologiche, che forniscono delle soluzioni «chiavi in mano», dei potenti riduttori d'incertezza (Downs 1957, 98-100). In questo caso le procedure referendarie finirebbero col perdere ogni ragion d'essere, dal momento che riprodurrebbero largamente quelle parlamentari. In Svizzera, tuttavia, ricerche su serie storiche di voti popolari hanno mostrato che la «lealtà» dei cittadini alle «consegne» dei partiti è assai relativa, tanto perché queste non sempre sono note, quanto perché considerate poco rilevanti (Kriesi 1994a, 67)! Se ne ricava che una dose d'imprevedibilità - le cui dimensioni variano ovviamente in base alle forze presenti, al contenuto delle questioni in discussione, ecc. - è effettivamente introdotta dai meccanismi referendari. Che ciò sia desiderabile o problematico conta poco. In questa sede ci si limita a sottolineare che, se così è, allora l'esercizio democratico non si esaurisce in una mera formalità. L'essenziale è che le procedure referendarie non siano un duplicato di quelle rappresentative: se da un lato, dunque, non è certa la loro funzione di contrappeso, dall'altro non si può neanche concludere in modo definitivo che esse si limitano a riprodurre la volontà di chi è al potere.

Anche se la democrazia referendaria fornisce ai settori più 
deboli e meno rappresentati un'occasione per partecipare ai processi decisionali, stando così le cose non possiamo purtroppo escludere che ciò avvenga a spese di coloro che sono ancora più deboli e che non hanno alcuna voce in capitolo (ad esempio gli stranieri senza diritto di voto). L'ipotesi del cittadino individualista, preoccupato di evitare i costi delle politiche pubbliche non può essere scartata a priori e si sa quanto un atteggiamento di questo tipo possa costituire un serio ostacolo allo sviluppo di politiche redistributive da perseguire attraverso la tassazione. Su questa linea, Offe (1987) ritiene che la ragione principale del declino del sostegno allo Stato sociale sia il passaggio da una percezione dominante delle politiche sociali come beni pubblici ad una percezione più diffidente in termini di costi e benefici individuali. L'bomo oeconomicus utilitarista descritto dalle teorie della rational choice cesserebbe così di essere un'astrazione per diventare il modello contemporaneo dell'individuo calcolatore. Da qui, non è del tutto assurda l'idea che più si favorisce la partecipazione e più si rischia paradossalmente di favorire le esclusioni. La tendenza dei gruppi a proteggere i diritti acquisiti (Olson 1982) vale anche per gli individui, almeno fin dal momento in cui essi dispongono dell'opportunità di decidere senza dover delegare il loro potere e senza rischiare sanzioni ${ }^{17}$.

I meccanismi referendari possono rivelarsi quindi totalmente controproducenti in rapporto all'obiettivo di dare più potere agli esclusi. A contrario si può dire che per conseguire questo scopo, il sistema politico è talvolta obbligato ad aggirare i canali democratici, quando vuole promuovere politiche favorevoli a categorie socialmente emarginate. La politica svizzera di lotta

17 In California, per esempio, la «proposta 13», dal contenuto notoriamente antifiscale, ha fatto molto rumore (Danzinger 1980; Goodenough 1982). Lanciata dal miliardario Howard Jarvis, essa ha raccolto una quantità di firme senza precedenti (circa un milione e mezzo), puntando a una riduzione di oltre il $50 \%$ dell'imposta fondiaria, allora assai elevata (2,5\% sul capitale). L'approvazione, il 6 giugno 1978, è stata massiccia, senza che i cittadini avessero necessariamente consapevolezza delle conseguenze socio-economiche: 750.000 dipendenti dello Stato furono immediatamente licenziati (Mény 1978, 112-113). Il fatto è che non si tratta di un caso isolato, proprio perché $\mathrm{i}$ meccanismi referendari si prestano a favorire la manifestazione di reazioni simili. Di recente per esempio, sempre in California, il 59\% degli elettori hanno accolto la «proposta 187» che vieta l'accesso a scuole e ospedali degli immigrati clandestini. L'applicazione di questo provvedimento è stata tuttavia sospesa fino all'esame della sua costituzionalità: senza dubbio, simili «ripari» non risultano inutili di fronte a certe manifestazioni del «popolo sovrano»... 
contro l'AIDS, presa a modello in tutta Europa (Bütschi e Cattacin 1994), così come gli esperimenti recenti di distribuzione controllata delle droghe (Kübler 1994) sarebbero stati difficilmente proponibili in una situazione di minaccia costante di ricorso al referendum, assumendo spesso le campagne referendarie su questi problemi connotazioni di guerre di religione. È significativo, per esempio, che uno dei responsabili dell'Assistenza elvetica ai malati di AIDS abbia dichiarato a un gruppo di studenti che lo intervistavano che la Svizzera si era fatta meno audace in materia nel momento in cui il dibattito aveva cominciato a politicizzarsi. Non è possibile liquidare in un sol colpo tale argomento sulla base della sua connotazione strettamente tecnica, dal momento che non possiamo ignorare che all'amministrazione spetta un ruolo di avanguardia illuminata in questi campi, in stretta collaborazione con le associazioni professionali e con quelle delle persone interessate. Queste politiche sono legittimate in modo solo frammentario, sia che si tratti di una legittimazione tecnica che considera la loro efficacia, sia che si tratti della loro legittimazione sociale presso le categorie interessate e la cui fiducia e cooperazione sono indispensabili per la loro messa in opera. La legittimazione democratica tradizionale, nella forma di istanze elettive o di sanzione del corpo elettorale, fa loro difetto. Ora, il problema è che una forma di legittimazione non può esistere che $a$ spese dell'altra. Più potere agli esclusi a spese della democrazia, dunque, o più democrazia rinunciando a dare più potere agli esclusi? Un crudele dilemma...

\section{Osservazioni conclusive}

Valutare le funzioni dei meccanismi referendari non ha senso, dunque, se non si tiene conto del quadro complessivo del funzionamento della democrazia, di cui questi meccanismi costituiscono uno degli aspetti. A questo riguardo, mi pare indispensabile sottolineare che tutti i sistemi democratici oscillano nel loro funzionamento fra due estremi: tendono da una parte all'elitismo, dall'altra al populismo. Non si tratta di un giudizio di valore, ma solamente di sottolineare la fragilità, la precarietà e la variabilità degli equilibri democratici, così come le tensioni alle quali vanno soggetti ${ }^{18}$. Ho già avuto modo di rilevare, nelle

${ }_{18}$ Sul punto rinvio al mio lavoro sulla democrazia locale (Papadopoulos 1994a). 
pagine precedenti, la contingenza della soglia accettabile di responsiveness del sistema politico e, simmetricamente, del grado della sua autonomia ottimale in rapporto alla società civile.

La tensione fra elitismo e populismo ruota intorno agli interrogativi sui limiti della delega e, correlativamente, sulle frontiere dell'esercizio della cittadinanza: la questione del grado di inclusione-esclusione nei processi politici è qui centrale. In relazione a tale tensione, non si può affatto parlare di rotture, ma piuttosto di differenze di grado: così, gli accordi neocorporativi possono essere considerati più elitisti delle decisioni parlamentari, in quanto fatti a porte chiuse e senza alcuna legittimazione elettiva delle parti, come del resto, a sua volta, la decisione parlamentare godrà di una insufficiente legittimazione democratica per il sostenitore della democrazia diretta. Sul piano concettuale, questa tensione comprende una serie di altre contrapposizioni:

1) quella fra la pedagogia politica dall'alto ${ }^{19}$ e la mistica sociale dal basso, contrapposizione che Landowski (1989) mette in evidenza a partire da un'analisi dei discorsi politici; e quella fra la cogitazione intellettuale e l'interazione sociale che preoccupa Wildavsky (1979) per la condotta delle politiche pubbliche;

2) la contrapposizione sottolineata dalla teoria sistemica fra, da una parte, la priorità da dare alla qualità degli outputs e alla rapidità di reazione del sistema, a rischio di un cedimento verso l'attivismo volontario, e, dall'altra, la priorità alla ricettività degli inputs, a rischio di affondare nell'immobilismo per eccesso di prudenza: tutto ciò privilegia sia l'efficacia, sia la legittimità decisionale;

3) il dilemma evocato dalla teoria delle scelte pubbliche fra l'obiettivo di ridurre $\mathrm{i}$ costi di transazione e quello di ridurre $\mathrm{i}$ costi esterni, quand'anche i due tipi di costi varino in senso inverso.

Si constata dunque che, se il consenso sulla democrazia come condizione per l'esercizio legittimo del potere è sempre più grande, il grado di democratizzazione dei singoli processi, così come le forme democratiche più appropriate, suscitano controversie. Abbiamo potuto constatare, per esempio, che la dimensione democratizzante dei meccanismi referendari non è

${ }^{19} \mathrm{Ci}$ sarebbe ancora da dire sulla compatibilità problematica di questo lavoro di Aufklärung con le esigenze democratiche; basta pensare ai dilemmi della modernizzazione autoritaria (Hermet 1975) in certi paesi del Terzo mondo (Turchia, Iran, ecc.). 
esente da critiche, e che anche se si è dei ferventi sostenitori della democratizzazione delle decisioni, ciò non significa affatto che si consideri necessariamente lo sviluppo di tali meccanismi come la strada principale per arrivarci. Attualmente, per esempio, l'analisi dell'azione statale è molto preoccupata per lo sviluppo delle possibilità di feedback democratico come risposta alle decisioni delle autorità. E questo non tanto per preoccupazioni di natura etica, quanto piuttosto per le ragioni di governabilità e di considerazione della complessità sociale che si sono viste in queste pagine. Ora, le procedure e le istituzioni immaginate non sono affatto necessariamente quelle della democrazia referendaria. $\mathrm{E}$ in effetti, tra le altre cose, si è potuto dimostrare che queste tendono ad una riduzione drastica delle scelte, presentandole in modo dicotomico. A partire da una riflessione sui limiti del dirigismo inerenti l'autonomia dei differenti sistemi sociali che si mostrano capaci di neutralizzare le velleità centraliste e interventiste dello Stato (Willke 1992), la tendenza è, piuttosto che un passaggio alla democrazia diretta, quella della negoziazione e contrattazione delle politiche pubbliche, della delega di funzioni secondo il principio di sussidiarietà, del ricorso alla valutazione pluralistica delle misure prese, ecc. Questo modo di procedere, che mira attraverso vari mezzi a rafforzare e ampliare le fasi di riflessione nei processi decisionali, consiste nel costruire procedure e regole del gioco favorevoli ad un'autovalutazione critica delle proprie preferenze da parte degli attori stessi, grazie ad un reciproco apprendistato, a uno sviluppo dell'empatia e dell'autolimitazione. In sintesi, tali procedure e regole del gioco hanno in comune l'ambizione ad attivare le risorse morali dei protagonisti delle controversie politiche. Ora, non è sicuro che i meccanismi referendari conducano a questo, tenuto anche conto delle obiezioni sulla qualità dei dibattiti e sulle motivazioni dei comportamenti di voto.

\section{[Traduzione di Pietro Grilli di Cortona]}

\section{Riferimenti bibliografici}

Andreani, T. (1990), Démocratie représentative, démocratie délégative, démocratie directe, in «Critique Communiste», XCVIII, pp. 33-43. App, R. (1987), Initiativen und ibre Wirkungen auf Bundesebene seit 1974, in «Annuaire Suisse de Science Politique», pp. 189-206. 
Ashby, W.R. (1956), An Introduction to Cybernetics, London, Chapmann and Hall.

Auer, A. (1989), Le référendum et l'initiative populaire aux Etats-Unis, Bâle-Paris, Helbing \& Lichtenhahn - Economica.

Bachrach, P. e M. Baratz (1962), Two Faces of Power, in «American Political Science Review», LVI, pp. 947-952.

Barber, B. (1984), Strong Democracy: Participatory Politics for a New Age, Berkeley, University of California Press.

Bendor, J.B. (1985), Parallel Systems: Redundancy in Government, Berkeley, University of California Press.

Bobbio, N. (1984), Il futuro della democrazia, Torino, Einaudi.

Buchanan, J.M. e J. Tullock (1962), The Calculus of Consent. Logical Foundations of Constitutional Democracy, Ann Arbor, The University of Michigan Press.

Bütschi, D. e S. Cattacin (1994), Le modèle suisse du bien-être, Lausanne, Réalités Sociales.

Cohen, J. e J. Rogers (1992), Secondary Associations and Democratic Governance, in «Politics and Society», XX, pp. 393-472.

Cronin, T.E. (1989), Direct Democracy. The Politics of Initiative, Referendum, and Recall, Cambridge (Mass.), Harvard University Press.

Crozier, M. et al. (1975), The Crisis of Democracy, New York, New York University Press, trad. it. La crisi della democrazia, Milano, Angeli, 1977.

Crozier, M. e E. Friedberg (1981), L'acteur et le système, Paris, Seuil.

Dahl, R.A. (1985), A Preface to Economic Democracy, Berkeley, University of California Press.

Danzinger, J.N. (1980), California's Proposition 13 and the Fiscal Limitation Movement in the US, in «Political Studies», XXVIII.

Delley, J.D. (1978), L'initiative populaire en Suisse, Lausanne, L'Age d'Homme.

Deutsch, K.W.D. (1976), Die Schweiz als ein paradigmatischer Fall politischer Integration, Berne, P. Haupt.

Dobry, M. (1986), Sociologie des crises politiques, Paris, Presses de la Fondation Nationale des Sciences Politiques.

Epple-Gass, R. (1991), Neue Formen politischer Mobilisierung: (k)eine Herausforderung der schweizerischen Demokratie?, in «Annuaire Suisse de Science Politique», pp. 151-171.

Gaxie, D. (1978), Le cens caché, Paris, Seuil.

Germann, R.E. et al. (1985), Experts et commissions de la Confédération, Lausanne, Presses polytechniques romandes.

Giugni, M. (1991), Les impacts de la démocratie directe sur les nouveaux mouvements sociaux, in «Annuaire Suisse de Science Politique», pp. 173-185.

Giugni, M. e H. Kriesi (1990), Nouveaux mouvements sociaux dans les années 80: évolution et perspectives, in «Annuaire Suisse de Science Politique», pp. 79-100. 
Goodenough, R. (1982), Proposition 13 and its Impact on Local Government Programmes in California, in «Policy and Politics», X, pp. 439-458.

Habermas, J. (1973), La technique et la science comme «idéologie», $\mathrm{Pa}$ ris, Gallimard.

- (1989), Ist der Herzschlag der Revolution zum Stillstand gekommen? Volkssouveränitat als Verfabren. Ein mormativer Begriff der Oeffentlichkeit?, in Forum für Philosophie Bad Homburg (Herausgeber), Die Ideen von 1789 in der deutschen Rezeption, Francoforte, Suhrkamp, pp. 7-36.

Hermet, G. (1975), Dictature bourgeoise et modernisation conservatrice: problèmes méthodologiques de l'analyse des situations autoritaires, in «Revue Française de Science Politique», XXV, pp. 10291061.

Hertig, H.P. (1982), Sind Abstimmungserfolge käuflich? Elemente der Meinungsbildung bei eidgenössischen Abstimmungen, in «Annuaire Suisse de Science Politique», pp. 35-57.

Immergut, E.M. (1992), Health Politics: Interests and Institutions in Western Europe, Cambridge (Mass.), Cambridge University Press.

Katzenstein, P.J. (1985), Small States in World Markets, Ithaca, Cornell University Press.

Kriesi, H. (1994a), Le défi à la démocratie directe posé par les transformations de l'éspace public, in Y. Papadopoulos (a cura di), Present et avenir de la démocratie directe, Genève, Georg, pp. 31-72.

- (1994b), Social Movements and Direct Democracy, paper presentato al Congresso mondiale dell'IPSA, Berlino, agosto.

Kübler, D. (1994), L'Etat face à la toxicomanie. Action publique et contrat social, Lausanne, Travaux de l'Institut de Science Politique.

Landowski, E. (1989), La société réfléchie, Paris, Seuil.

Lavau, G. (1969), Le parti communiste dans le système politique français, in F. Bon (a cura di), Le communisme en France, Paris, A. Colin, Cahiers de la Fondation nationale des sciences politiques, pp. 7-81.

- (1986), L'électeur devient-il individualiste?, in P. Birnbaum e J. Lecat (a cura di), Sur l'individualisme, Paris, Presses de la Fondation Nationale des Sciences Politiques, pp. 301-329.

Lenoir, R. (1989), La genèse sociale d'un problème social, in P. Champagne et al., Initiation à la pratique sociologique, Paris, Dunod, pp.78-98.

Lijphart, A. (1977), Democracy in Plural Societies, New Haven, Yale University Press.

Macpherson, C. (1985), Principes et limites de la démocratie libérale, Paris-Montreal, La Découverte-Boréal Express.

Magleby, D.B. (1994), I problematici sviluppi della recente esperienza 
statunitense, in M. Caciagli e P.V. Uleri (a cura di), Democrazie e referendum, Roma-Bari, Laterza, pp. 79-99.

Mény, Y. (1978), Initiative populaire, référendum et «recall» dans les Etats américains, in «Pouvoirs», VII, pp. 107-113.

Neidhart, L. (1983), Regierbarkeitsfragen in der direkten Demokratie, in «Annuaire Suisse de Science Politique», pp. 13-43.

Offe, C. (1984), Contradictions of the Welfare State, London, Hutchinson.

- (1987), Democracy Against the Welfare State? Structural Foundations of Neoconservative Political Opportunities, in «Political Theory», XV, pp. 501-537.

- (1989), Bindung, Fessel, Bremse. Die Unübersichtlichkeit von Selbstbeschränkungsformeln, in A. Honneth et al., Zwischenbetrachtungen im Prozess der Aufklärung, Francfort, Suhrkamp, pp. 739-774.

Olson, M. (1982), The Rise and Decline of Nations, New Haven e London, Yale University Press, trad. it. (1984), Ascesa e declino delle nazioni, Bologna, Il Mulino.

Ossipow, W. (1988), Les groupes d'intérêt dans le processus de décision et la communication politique. Une interpretation systémique du cas suisse, in Jean Meynaud ou l'utopie revisitée, Lausanne, Université de Lausanne, pp. 109-125.

Papadopoulos, Y. (1991), Quel rôle pour les petits partis dans le démocratie directe?, in «Annuaire Suisse de Science Politique», 1991, pp. 131-150.

- (a cura di) (1994), Elites politiques et peuple en Suisse. Analyse des votations fédérales 1970-1987, Lausanne, Réalités sociales.

- (1994), Les tensions de la démocratie locale, in M. Bassand e J.P. Leresche (a cura di), Les faces cachées de l'urbain, Berne, P. Lang, pp. 139-153.

Pateman, C. (1970), Participation and Democratic Theory, Cambridge, Cambridge University Press.

Poulantzas, N. (1978), L'Etat, le pouvoir, le socialisme, Paris, PUF.

Ranney, A. (1994), Nuove pratiche e vecchia teoria, in M. Caciagli e P.V. Uleri (a cura di), Democrazie e referendum, Roma-Bari, Laterza, pp. 29-48.

Rose, R. (1980), Challenge to Governance, London, Sage.

Saris, W. (1991), New Possibilities for Political Participation: TeleDemocracy, in «Annuaire Suisse de Science Politique», pp. 327336.

Sartori, G. (1976), Parties and Party Systems, Cambridge, Cambridge University Press.

- (1987), The Theory of Democracy Revisited, Chatham, Chatham House Publishers.

Scharpf, F.W. (1990), La social-démocratie face à la crise, Paris, Economica. 
- (1991), Political Institutions, Decision Styles and Policy Choices, in R. Czada e A. Windhoff-Héritier (a cura di), Political Choice, Francfort-Boulder, Campus-Westview Press.

Schmidt, M.G. (1982), The Role of the Parties in Shaping Macroeconomic Policy, in F.G. Castles (a cura di), The Impact of Parties, London, Sage, pp. 97-176.

Schumpeter, J. (1964), Capitalismo, socialismo, democrazia, Milano, Etas Kompass.

Sciarini, P. (1992), La Suisse dans la négociation sur l'Espace économique européen: de la rupture à l'apprentissage, in «Annuaire Suisse de Science Politique», pp. 297-322.

Smith, G. (1986), The Functional Properties of the Referendum, in «European Journal of Political Research», IV, pp. 1-23.

Suksi, M. (1993), Bringing in the People. A Comparison of Constitutional Forms and Practices of the Referendum, Dordrecht, Martinus Nijhoff.

Tarrow, S. (1988), Struggle, Politics, and Reform, Ithaca, Cornell University Press.

Uleri, P.V. (1994), The Referendum Phenomenon in Italy: from the Beginning to the Crisis of a Democratic System, paper ECPR, Madrid.

Weber, M. (1968), Economia e società, Milano, Comunità, 2 voll.

Wildavsky, A.B. (1979), Speaking Truth to Power. The Art and Craft of Policy Analysis, Boston, Little Brown.

Willke, H. (1992), Ironie des Staates, Francfort, Suhrkamp.

Zolo, D. (1992), Il principato democratico, Milano, Feltrinelli. 